\title{
High-pressure microfluidics
}

\author{
K. Hjort \\ The Microsystems Technology Division, Department of Engineering, \\ The Angstrom Laboratory, Uppsala University, 75121 Uppsala, Sweden
}

\begin{abstract}
When using appropriate materials and microfabrication techniques, with the small dimensions the mechanical stability of microstructured devices allows for processes at high pressures without loss in safety. The largest area of applications has been demonstrated in green chemistry and bioprocesses, where extraction, synthesis and analyses often excel at high densities and high temperatures, which are accessible through high pressures. Capillary chemistry has been used since long but, just like in low-pressure applications, there are several potential advantages in using microfluidic platforms, e.g., planar isothermal set-ups, large local variations in geometries, dense form factors, small dead volumes and precisely positioned microstructures for control of reactions, catalysis, mixing and separation. Other potential applications are in, e.g., microhydraulics, exploration, gas driven vehicles, and high-pressure science. From a short review of the state-of-art and frontiers of high pressure microfluidics, the focus will be on our different solutions demonstrated for microfluidic handling at high pressures and challenges that remain.
\end{abstract}

